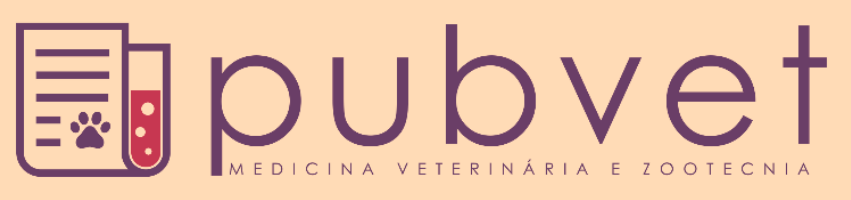

https://doi.org/10.31533/pubvet.v15n02a745.1-9

\title{
Geoestatística bidimensional como alternativa à modelagem de séries temporais sob cenários de mudanças climáticas
}

\author{
Vania Corrêa Mota ${ }^{*} *$

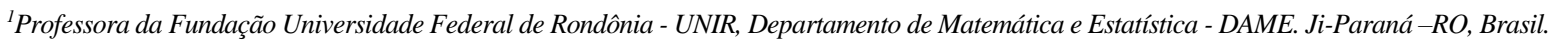 \\ *Autora para correspondência, E-mail: vaniamota33@gmail.com
}

\begin{abstract}
Resumo. O objetivo deste trabalho foi verificar o uso de modelos de semivariograma e krigagem ordinária para caracterizar e representar a dependência temporal da precipitação pluvial e da temperatura do ar, utilizando anos e meses como coordenadas referenciadas. Foram utilizados os dados de previsão de precipitação pluvial e temperatura do ar em cenários futuros de mudanças climáticas, de 2000 a 2099, do município de Juiz de Fora, MG. Com base nos semivariogramas experimentais e teóricos, considerando isotropia e anisotropia, foi constatada dependência temporal da precipitação pluvial, com melhor ajuste do modelo "wave", quando comparado com os modelos gaussiano e esférico. A estrutura e a magnitude de dependência temporal das duas variáveis, em cenários futuros e nas direções $0^{\circ}$ (meses) e $90^{\circ}$ (anos), foram caracterizadas utilizando-se o arranjo proposto. Com o uso da geoestatística, o padrão da variabilidade temporal da precipitação pluvial e da temperatura do ar foi representado ao longo dos anos e dos meses por meio da interpolação de krigagem, verificando-se possíveis mudanças nos padrões de precipitação pluvial e temperatura, para os dados de previsão de mudanças climáticas, com maior alteração para o cenário mais pessimista.
\end{abstract}

Palavras-chave: Geoestatística, temperatura, precipitação, variabilidade temporal

\section{Bidimensional geostatistics as an alternative for modeling time series under climate change scenarios}

\begin{abstract}
The objective of this work was to verify the use of semivariogram models and ordinary kriging to characterize and to represent the temporal dependence of the pluvial precipitation and air temperature, by using years and months with coordinates. The observed the forecast data of future scenarios of climatic changes, from 2000 to 2099, of the municipal district of Juiz de Fora-MG, for the pluvial precipitation and air temperature were analyzed. The semivariograms were adjusted by considering isotropy and anisotropy conditions. The wave model presented better adjustment than the Gaussian and spherical models when the two-dimensional grid was used. The structure and the magnitude of temporal dependence of the two variables were characterized by considering the $0^{\circ}$ (months) and $90^{\circ}$ (years) directions. The pattern of the temporal variability of the precipitation and the air temperature was represented by using kriging. Possible changes in the patterns of the pluvial precipitation and the air temperature for the future scenarios were verified by using a two-dimensional grid and kriging. The greatest changes in the pattern of the two variables were verified in the most pessimist future scenario.
\end{abstract}

Keywords: Geostatistics, temperature, precipitation, temporal variability 


\title{
Geoestadística como alternativa para la modelación bidimensional de series temporales bajo escenarios de cambios climáticos
}

\begin{abstract}
Resumen. El objetivo de este estudio fue investigar el uso de diferentes modelos de semivariograma y kriging ordinario para caracterizar y representar la dependencia temporal de lluvia y da temperatura del aire, usando años y meses como coordenadas de referencia. Se utilizó los datos de pronóstico de precipitación y temperatura del aire en futuros escenarios de cambio climático de 2000 hasta 2099, para el municipio de Juiz de Fora MG. Con base en los semivariogramas experimentales y los teóricos que consideran isotropía y anisotropía, se constató la dependencia temporal de la precipitación, con el mejor modelo de "wave" en comparación con los modelos esféricos y gaussianos. La estructura y la magnitud de la dependencia temporal de las dos variables en los escenarios futuros en las direcciones $0^{\circ}$ (meses) hasta $90^{\circ}$ (años) fueron caracterizadas utilizando el ajuste propuesto. Con el uso de la geoestadística, el patrón de la variabilidad temporal de la precipitación y temperatura del aire fue representado con el paso de los años y de los meses por medio de la interpolación de kriging, observando posibles cambios en los patrones de precipitación y temperatura para los datos de previsión de los cambios climáticos, con gran alteración en el peor de los casos.
\end{abstract}

Palabras clave: Geoestadística, temperatura, precipitación, variabilidad temporal

\section{Introdução}

Muitos pesquisadores das áreas de climatologia, meteorologia, hidrologia e áreas afins têm verificado a ocorrência de alterações e mudanças climáticas em diversas regiões do planeta.

Algumas dessas alterações são verificadas quando se pretende modelar elementos climáticos, como dados de precipitação pluvial e temperatura do ar. Para estudar a precipitação pluvial e a temperatura do ar, é necessário caracterizar a distribuição dessas variáveis ao longo do tempo. No entanto, de posse de extensas séries históricas, torna-se mais complexa a identificação do comportamento espaço-temporal dos dados. Muitos fatores afetam essa distribuição e isso determina sua variação espacial, temporal e sazonal (Mendonça \& Danni-Oliveira, 2017). Para estudar essa variação, estão disponíveis, nos dias atuais, muitas técnicas de interpolação espacial com variados graus de complexidade, de forma a representar processos climatológicos, tal como a metodologia geoestatística (Goovaerts, 1997; Mendonça \& Danni-Oliveira, 2017).

A análise geoestatística não se limita à obtenção de um modelo de dependência espacial ou temporal, desejando-se também predizer valores em pontos não amostrados. O interesse pode ser em um ou mais pontos específicos da área ou em obter uma malha de pontos interpolados que permitam visualizar o comportamento da variável na região por meio de um mapa que descreva melhor a variabilidade espacial e ou temporal dos dados. Fenômenos naturais apresentam-se frequentemente estruturados com relação à vizinhança. Dessa forma, pode-se dizer que essas variações não são aleatórias e, portanto, apresentam algum grau de dependência espacial ou temporal.

Fisicamente, existe uma diferença entre as dimensões espaciais e temporais, pois as unidades das coordenadas dos dois processos apresentam grandezas diferentes e os modelos precisam considerá-las (Chilès \& Delfiner, 2009). Para contornar esse problema, utiliza-se um arranjo temporal proposto por Sant'Anna Neto (2005) e utilizada por Mota (2008) e Mota et al. (2008), no qual consideram-se anos e meses como se fossem coordenadas de longitude e latitude, respectivamente, para caracterizar a dependência temporal de variáveis climáticas, tais como a precipitação pluvial e a temperatura.

Assim, objetivou-se com o presente trabalho, verificar o uso de modelos de semivariograma e utilizar a krigagem ordinária para caracterizar e representar a dependência temporal da precipitação pluvial e da temperatura, para os cenários futuros de mudanças climáticas, utilizando anos e meses como coordenadas. 


\section{Material e Métodos}

Este estudo foi realizado com dados do município de Juiz de Fora, estado de Minas Gerais. Segundo a classificação internacional de Köppen, o clima da região é do tipo tropical de altitude CWA, caracterizado por duas estações bem definidas: uma seca e de menores temperaturas, que se estende de maio a setembro, e outra úmida e de temperaturas mais elevadas, de outubro a abril. A temperatura média anual é de $19,3^{\circ} \mathrm{C}$ e a precipitação anual é de $1.644 \mathrm{~mm}$ (Brasil, 1992).

Os dados de previsão de cenários futuros, referem-se as variáveis: precipitação pluvial mensal, em $\mathrm{mm}$ e temperatura média do ar, em ${ }^{\circ} \mathrm{C}$. Esses dados de previsão de cenários futuros foram obtidos da base de dados do 'Intergovernmental Panel on Climate Change' - IPCC (IPCC, 2001) no período de 2000 a 2099. Esses dados fazem parte de previsões realizadas pelo 'National Center for Atmospheric Research' (NCAR, 2007), referentes ao Terceiro Relatório de Avaliação (TAR), modelo CCSM 3.0, com a resolução espacial de $1,4^{\circ}$ x $1,4^{\circ}$, com latitude de $-21,7119^{\circ}$ e longitude de $-43,5938^{\circ}$. Para efeito de análise, esse período foi dividido em três: P1, de 2000 a 2032; P2, de 2033 a 2065 e P3, de 2066 a 2099 (para a precipitação) ou de 2066 a 2098 (para a temperatura). Optou-se em utilizar um cenário mais pessimista (A2), caracterizado por um mundo futuro muito heterogêneo onde a regionalização é dominante; um intermediário (A1B), onde os indivíduos procuram riqueza pessoal em lugar de qualidade ambiental e outro mais otimista (B1), caracterizado por rápida mudança na estrutura econômica mundial, com introdução de tecnologias limpas.

Os semivariogramas foram estimados por meio dos estimadores clássico de Matheron, conforme Eq. 1 citado por Mota et al. (2019):

$\gamma(h)=\frac{1}{2 N(h)} \sum_{j=1}^{N(h)}\left[z\left(x_{j}\right)-z\left(x_{j}+h\right)\right]^{2}$,

em que $N(h)$ é o número de pares possíveis para a distância $h, \hat{\gamma}(h)$ é a semivariância para uma distância $h, z\left(x_{j}\right)$ e $z\left(x_{j}+h\right)$ são as observações de precipitação pluvial e temperatura separadas pelo vetor $h$ e $h$ é a distância de separação das observações, nesse caso, o tempo. Nas análises como as observações foram coletadas ao longo do tempo, consideraram-se, no arranjo proposto, anos e meses como coordenadas referenciadas, conforme proposta por Sant'Anna Neto (2005) e Mota et al. (2008) da mesma forma como longitude e latitude são utilizadas no caso de dados georreferenciados. Para o ajuste do semivariograma teórico, foi utilizado o método dos mínimos quadrados ordinários (OLS). Os modelos estatísticos isotrópicos comparados foram: esférico, gaussiano e 'wave', que conforme descrito em Goovaerts (1997) e Mota et al. (2008) são definidos por:

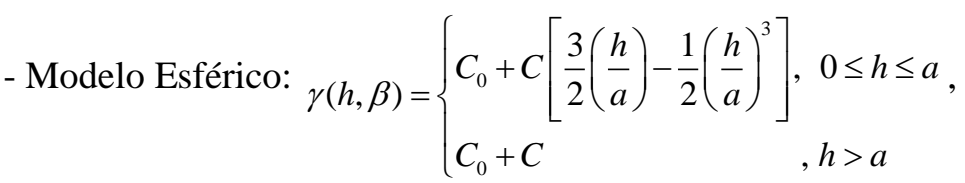

-Modelo Gaussiano: $\gamma(h, \beta)=C_{o}+C_{1}\left(1-\exp \left(-3\left(\frac{h^{2}}{a^{2}}\right)\right)\right)$, se $a>0$,

- Modelo 'wave': $\gamma(h, \beta)=C_{0}^{2}+\frac{a}{h} \operatorname{sen}\left(\frac{h}{a}\right)$,

em que $\gamma(h, \beta)$ é a semivariância para a distância $h ; C_{o}$ é o efeito pepita; $C_{o}+C_{l}$ é o patamar; $a$ é o alcance da dependência espacial.

Do conjunto de resultados obtidos, pelos estimadores clássico, para os três modelos, foram estimados seus parâmetros e selecionado o melhor modelo, de acordo com a aproximação para o valor do Critério de Akaike (AIC), Eq. 5, proposta por Webster \& Oliver (2001), ou seja:

$A I C=2 p+n \cdot \ln (R S S / n)$

em que $n$ é o número de observações, $p$ é o número de parâmetros e $R S S$ é a soma de quadrados de 
resíduos. A relação $\mathrm{Co} / \mathrm{Co}+\mathrm{C}_{1}$ foi utilizada para avaliar o grau de dependência espacial das variáveis e como, neste estudo, os dados são analisados ao longo do tempo, essa relação foi chamada de grau de dependência temporal, adaptada de Cambardella et al. (1994).

Para realizar a interpolação por krigagem ordinária e mapear as variáveis em estudo, utilizaram-se às estimativas dos parâmetros referentes ao melhor modelo de semivariograma selecionado anteriormente. De acordo com Goovaerts (1997), o estimador linear é dado pela seguinte equação:

$$
Z_{K O}^{*}(x)=\sum_{j=1}^{n(x)} \lambda_{j}(x) z\left(x_{j}\right)
$$

em que $n(x)$ é o número de observações de $z\left(x_{j}\right)$ utilizadas para estimar $Z_{K O}^{*}(x)$ e $\lambda_{j}$ são os pesos associados às observações $z\left(x_{j}\right)$. Nesse caso, para que $Z_{K O}^{*}(x)$ seja não tendencioso, a soma dos pesos das observações deve ser igual a $1 \mathrm{e}$, a esperança entre o valor estimado e o observado dever ser zero e a variância da estimativa deve ser mínima, respectivamente.

A metodologia de interpolação por krigagem ordinária foi utilizada para gerar uma superfície suavizada dos mapas de contorno. Para verificar se a krigagem ordinária descreve adequadamente a variabilidade temporal das variáveis analisadas, foi utilizada a validação cruzada ("cross-validation") (Mota, 2008; Mota et al., 2008). Como critérios para a validação cruzada, foram considerados o erro médio (EM), e a razão do quadrado médio do erro (RQME) (Soares et al., 2017), definidos respectivamente por:

$$
\begin{aligned}
& \mathrm{EM}=\frac{1}{n} \sum_{j=1}^{n}\left[z\left(x_{j}\right)-\hat{z}\left(x_{j}\right)\right] \approx 0, \\
& \mathrm{RQME}=\left\{\frac{1}{n} \sum_{j=1}^{n}\left[\frac{z\left(x_{j}\right)-\hat{z}\left(x_{j}\right)}{\sigma_{E}\left(x_{j}\right)}\right]^{2}\right\}^{\frac{1}{2}} \approx 1 .
\end{aligned}
$$

Espera-se que o EM seja próximo de zero e o RQME seja próximo de 1 (Webster \& Oliver, 2001). Todas as análises foram realizadas no pacote geoR (Ribeiro Júnior \& Diggle, 2001), do ambiente computacional R (R-Core-Team, 2016).

\section{Resultados e discussão}

Para os cenários futuros A2, A1B e B1, nos períodos P1, P2 e P3, verificou-se dependência temporal da precipitação pluvial e da temperatura do ar, pois o valor absoluto da diferença entre as observações aumentou com o aumento da distância até um ponto de estabilidade que separa o universo estruturado do aleatório, correspondente ao alcance, de forma a satisfazer as suposições de estacionaridade.

De acordo com a aproximação do critério de informação de Akaike para os cenários A2, A1B e B1, observou-se melhor ajuste do modelo "wave" à série de precipitação pluvial e aos dados de temperatura do ar, para os três períodos em estudo, conforme pode ser observado nas Tabelas 1 e 2 respectivamente.

Os dados de precipitação pluvial e temperatura do ar para a cidade de Juiz de Fora são candidatos naturais para ajuste do modelo "wave", por serem periódicos. Entretanto, os semivariogramas obtidos pelo modelo "wave" não são restritos a apenas uma estrutura monotônica. Este modelo também pode apresentar segmentos cíclicos ou decrescentes, os quais são chamados de efeito buraco ou "holle effect" (Andriotti, 2003). Isso pode ser um dos principais motivos que determinaram melhor ajuste do modelo "wave" aos cenários A2, A1B e B1. Ignorar essas estruturas pode resultar em modelos não realísticos, os quais não reproduzem as variabilidades espaciais e temporais observadas.

Nos mapas de krigagem isotrópicos para os cenários futuros de mudanças climáticas, apresentados na Figura 1, pode ser verificada a variabilidade temporal da precipitação pluvial e da temperatura do ar, em que são visíveis os principais padrões da variável, caracterizados por áreas de maior e menor concentração das chuvas e as áreas com altas e baixas temperaturas. 
Tabela 1. Estimativa dos parâmetros efeito pepita $\left(\mathrm{C}_{0}\right)$, patamar $\left(\mathrm{C}_{0}+\mathrm{C}_{1}\right)$, alcance $(a)$, critério de Akaike (AIC) e grau de dependência temporal (DT) dos modelos esférico (Esf.), gaussiano (Gaus) e "wave" (Wav) ajustados aos semivariogramas experimentais clássico relativos à precipitação pluvial mensal de Juiz de Fora, para o cenário A2, A1B e B1 nos três períodos.

\begin{tabular}{|c|c|c|c|c|c|c|c|c|c|}
\hline & \multicolumn{9}{|c|}{ Estimador clássico } \\
\hline & \multicolumn{3}{|c|}{ Cenário A2 P1 } & \multicolumn{3}{|c|}{ Cenário A2 P2 } & \multicolumn{3}{|c|}{ Cenário A2 P3 } \\
\hline & Esf. & Gaus & Wav & Esf. & Gaus & Wav & Esf. & Gaus & Wav \\
\hline$a$ & 7,08 & 5,84 & 7,51 & 7,08 & 5,83 & 7,47 & 7,14 & 5,83 & 7,56 \\
\hline$C_{0}$ & 0,00 & 296,31 & 585,00 & 0,00 & 250,95 & 562,67 & 0,00 & 250,954 & 654,92 \\
\hline$C_{0}+C_{1}$ & 3838,74 & 3554,84 & 3144,53 & 4203,18 & 3966,03 & 3513,88 & 4544,04 & 3966,03 & 3756,69 \\
\hline AIC & 3163,13 & 3135,58 & 2840,98 & 3259,32 & 3220,31 & 2911,67 & 3300,04 & 3220,31 & $\mathbf{3 0 8 1 , 5 5}$ \\
\hline \multirow[t]{3}{*}{ DT } & $0,00 \%$ & $8,33 \%$ & $18,60 \%$ & $0,00 \%$ & $6,00 \%$ & $16,01 \%$ & $0,00 \%$ & $6,00 \%$ & 17,43 \\
\hline & \multicolumn{3}{|c|}{ Cenário A1B P1 } & \multicolumn{3}{|c|}{ Cenário A1B P2 } & \multicolumn{3}{|c|}{ Cenário A1B P3 } \\
\hline & Esf. & Gaus & Wav & Esf. & Gaus & Wav & Esf. & Gaus & Wav \\
\hline$a$ & 7,14 & 5,86 & 7,47 & 7,11 & 5,83 & 7,51 & 6,91 & 5,69 & 7,33 \\
\hline$C_{0}$ & 0,00 & 282,58 & 593,66 & 0,00 & 283,90 & 637,87 & 0,00 & 288,97 & 590,27 \\
\hline$C_{0}+C_{1}$ & 4225,23 & 3956,29 & 3504,66 & 4514,25 & 4245,38 & 3744,50 & 4232,22 & 3958,92 & 3515,20 \\
\hline AIC & 3248,93 & 3213,26 & 2922,47 & 3294,95 & 3256,55 & 2995,67 & 3382,23 & 3351,53 & 2993,52 \\
\hline \multirow[t]{3}{*}{ DT } & $0,00 \%$ & 7,14 & $16,94 \%$ & $0,00 \%$ & $8,72 \%$ & $17,03 \%$ & $0,00 \%$ & $7,29 \%$ & $16,79 \%$ \\
\hline & \multicolumn{3}{|c|}{ Cenário B1 P1 } & \multicolumn{3}{|c|}{ Cenário B1 P2 } & \multicolumn{3}{|c|}{ Cenário B1 P3 } \\
\hline & Esf. & Gaus & Wav & Esf. & Gaus & Wav & Esf. & Gaus & Wav \\
\hline$a$ & 7.17 & 5,93 & 7,51 & 7,05 & 5,79 & 7,33 & 7,08 & 5,93 & 7,42 \\
\hline$C_{0}$ & 0,00 & 331,55 & 633,09 & 0,00 & 307,16 & 639,97 & 0,00 & 262,21 & 594,77 \\
\hline$C_{0}+C_{1}$ & 4219,52 & 3901,69 & 3455,22 & 4375,37 & 4082,58 & 3604,05 & 4406,92 & 4159,56 & 3677,01 \\
\hline AIC & 3206,85 & 3173,23 & 2941,80 & 3254,62 & 3221,33 & 2958,70 & 3378,71 & 3337,50 & 3057,08 \\
\hline DT & $0,00 \%$ & $8,49 \%$ & $18,32 \%$ & $0,00 \%$ & $7,52 \%$ & $17,75 \%$ & $0,00 \%$ & $6,30 \%$ & $16,17 \%$ \\
\hline
\end{tabular}

Obs.: Cenário mais pessimista (A2), cenário intermediário (A1B) e cenário otimista (B1), nos períodos de 2000 a 2032 (P1), 2033 a $2065(\mathrm{P} 2)$ e 2066 a 2099 (P3).

Tabela 2. Estimativa dos parâmetros efeito pepita $\left(\mathrm{C}_{0}\right)$, patamar $\left(\mathrm{C}_{0}+\mathrm{C}_{1}\right)$, alcance $(a)$, critério de Akaike (AIC) e grau de dependência temporal (DT) dos modelos esférico (Esf.), gaussiano (Gaus) e "wave" (Wav) ajustados aos semivariogramas experimentais clássico relativos à temperatura do ar de Juiz de Fora, para o cenário A2, A1B e B1 nos três períodos.

\begin{tabular}{|c|c|c|c|c|c|c|c|c|c|}
\hline & \multicolumn{9}{|c|}{ Estimador clássico } \\
\hline & \multicolumn{3}{|c|}{ Cenário A2 P1 } & \multicolumn{3}{|c|}{ Cenário A2 P2 } & \multicolumn{3}{|c|}{ Cenário A2 P3 } \\
\hline & Esf. & Gaus & Wav & Esf. & Gaus & Wav & Esf. & Gaus & Wav \\
\hline$a$ & 5,05 & 4,13 & 5,67 & 4,90 & 4,03 & 5,62 & 4,71 & 3,94 & 5,44 \\
\hline$C_{0}$ & 0.00 & 0,38 & 1,00 & 0,00 & 0,37 & 1,00 & 0,00 & 0,42 & 0,67 \\
\hline$C_{0}+C_{1}$ & 5,37 & 5,00 & 4,33 & 4,96 & 4,60 & 3,93 & 4,44 & 4,02 & 3,73 \\
\hline AIC & $-1618,01$ & $-1618,30$ & $-1959,47$ & $-1674,14$ & $-1673,47$ & $-1996,26$ & $-1786,79$ & -1782.50 & $-2057,62$ \\
\hline \multirow[t]{3}{*}{ DT } & $0,00 \%$ & $7,60 \%$ & $23,09 \%$ & $0,00 \%$ & $8,04 \%$ & $25,44 \%$ & $0,00 \%$ & $10,44 \%$ & $17,96 \%$ \\
\hline & \multicolumn{3}{|c|}{ Cenário A1B P1 } & \multicolumn{3}{|c|}{ Cenário A1B P2 } & \multicolumn{3}{|c|}{ Cenário A1B P3 } \\
\hline & Esf. & Gaus & Wav & Esf. & Gaus & Wav & Esf. & Gaus & Wav \\
\hline$a$ & 4,91 & 4,04 & 5,53 & 4,86 & 4,01 & 5,49 & 4,70 & 3,94 & 5,49 \\
\hline$C_{0}$ & 0,00 & 0,36 & 0,61 & 0,00 & 0,37 & 0,61 & 0,00 & 0,38 & 0,59 \\
\hline$C_{0}+C_{1}$ & 4,94 & 4,58 & 4,29 & 4,72 & 4,35 & 4,07 & 4,15 & 3,77 & 3,53 \\
\hline AIC & $-1686,71$ & $-1686,50$ & $-2036,61$ & $-1702,70$ & $-1700,91$ & $-2015,85$ & $-1807,91$ & $-1803,10$ & $-2143,10$ \\
\hline \multirow[t]{2}{*}{ DT } & $0,00 \%$ & $7,86 \%$ & $14,22 \%$ & $0,00 \%$ & $8,50 \%$ & $14,98 \%$ & $0,00 \%$ & $10,07 \%$ & $16,71 \%$ \\
\hline & \multicolumn{3}{|c|}{ Cenário B1 P1 } & \multicolumn{3}{|c|}{ Cenário B1 P2 } & \multicolumn{3}{|c|}{ Cenário B1 P3 } \\
\hline & Esf. & Gaus & Wav & Esf. & Gaus & Wav & Esf. & Gaus & Wav \\
\hline$a$ & 4,94 & 4,04 & 5,58 & 4,86 & 4,01 & 5,53 & 4,71 & 3,92 & 5,49 \\
\hline$C_{0}$ & 0,00 & 0,34 & 0,60 & 0,00 & 0,33 & 0,57 & 0,00 & 0,38 & 0,63 \\
\hline$C_{0}+C_{1}$ & 5,13 & 4,80 & 4,49 & 4,78 & 4,45 & 4,17 & 4,54 & 4,16 & 3,88 \\
\hline AIC & $-1633,06$ & $-1633,95$ & $-1981,53$ & $-1662,55$ & $-1662,17$ & $-1985,97$ & $-1730,86$ & $-1727,42$ & $-2049,41$ \\
\hline DT & $0,00 \%$ & $7,00 \%$ & $13,36 \%$ & $0,00 \%$ & $7,41 \%$ & $13,67 \%$ & $0,00 \%$ & $9,13 . \%$ & $16,24 \%$ \\
\hline
\end{tabular}

Obs.: Cenário mais pessimista (A2), cenário intermediário (A1B) e cenário otimista (B1), nos períodos de 2000 a 2032 (P1), 2033 a 2065 (P2) e 2066 a 2098 (P3).

Com base nos mapas de krigagem da precipitação e da temperatura, observou-se tendência de menores índices de precipitação concentrados nos meses de maio a setembro, com temperaturas mais baixas. Já os índices mais elevados de precipitação se concentraram nos meses de outubro a abril, com temperaturas mais altas. Isso ocorre de acordo com o padrão climático local, pois os dados de 
precipitação e de temperatura avaliados são referentes a uma região geográfica com o período seco e chuvoso bem definido, conforme relatado por Marengo et al. (2007). No período chuvoso, a precipitação apresentou valores máximos próximos a $250 \mathrm{~mm} / \mathrm{mês}$; (Figura 1) já no período seco, o índice pluvial apresentou valores mínimos próximos de zero, para os três cenários.
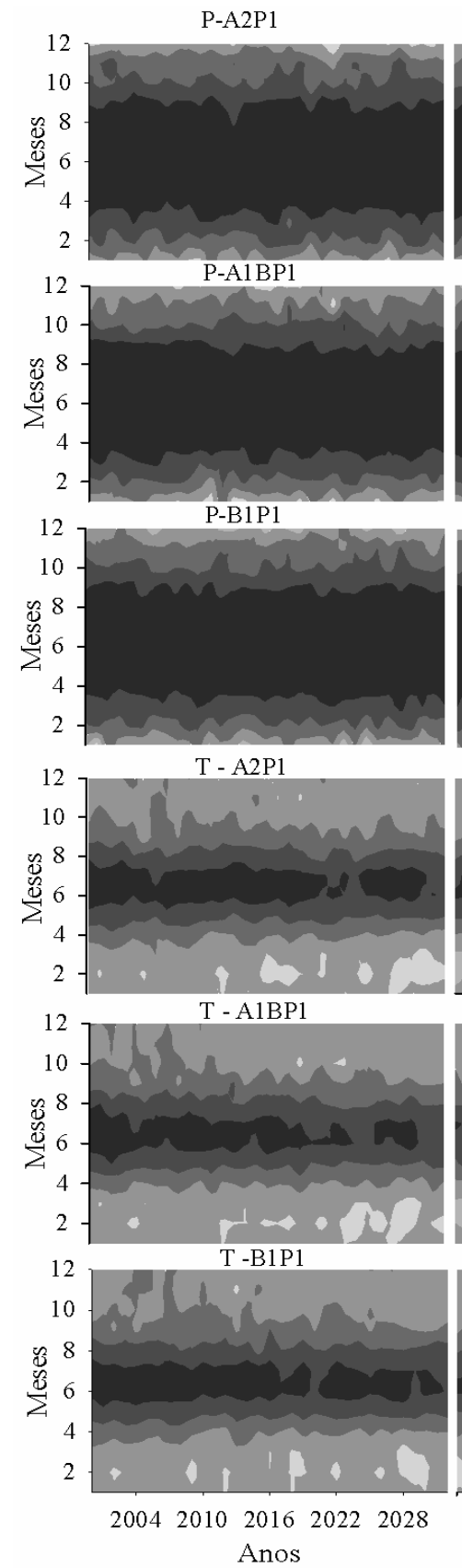

P-A2P2

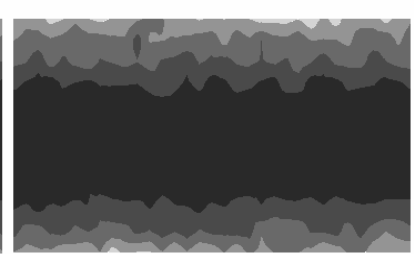

P-A1BP2

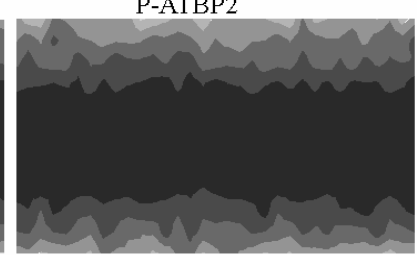

P-B1P2

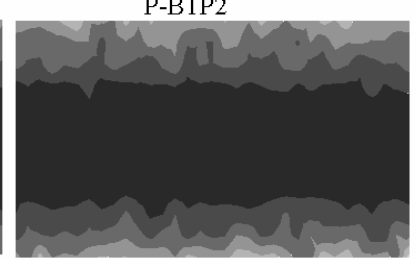

$\mathrm{T}-\mathrm{A} 2 \mathrm{P} 2$

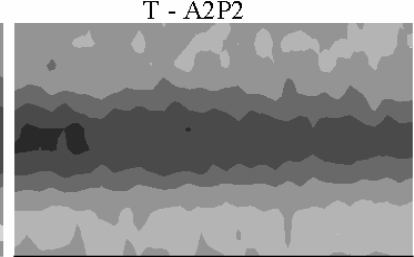

T - A1BP2

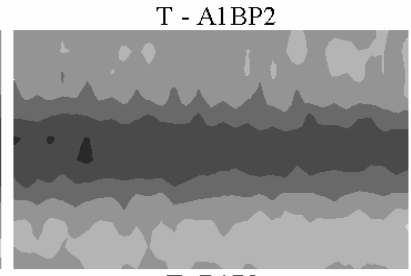

$\mathrm{T}-\mathrm{B} 1 \mathrm{P} 2$

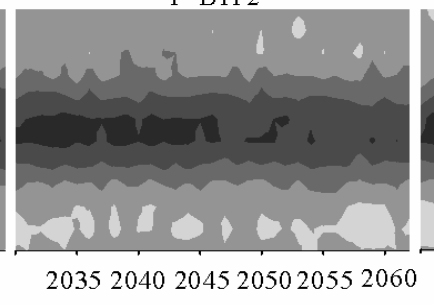

P-A2P3

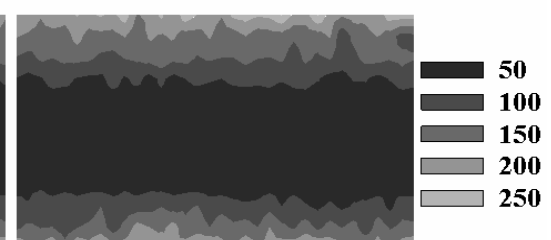

P-A1BP3

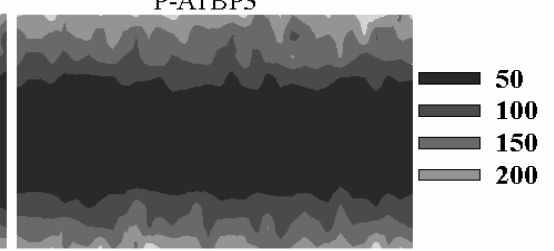

P-B1P3

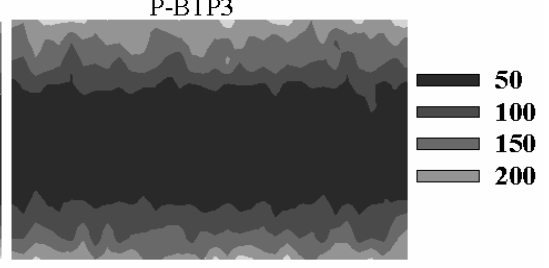

$\mathrm{T}-\mathrm{A} 2 \mathrm{P} 3$
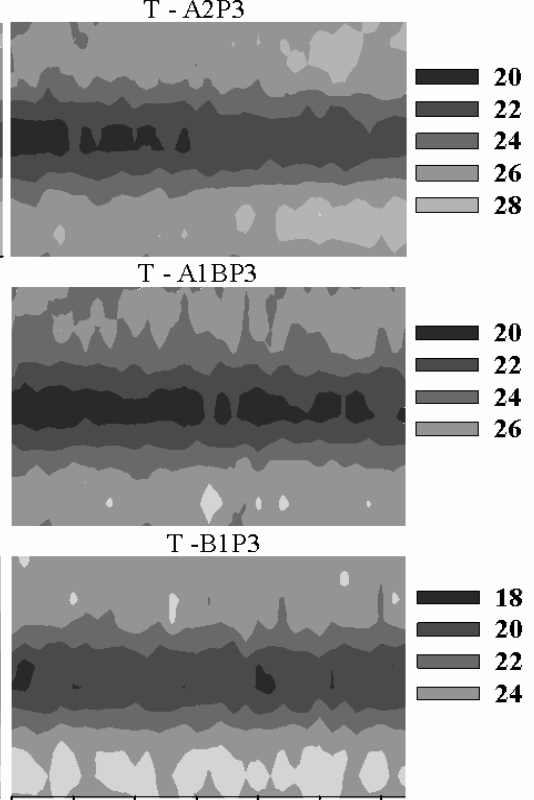

Figura 1. Mapas de krigagem da precipitação pluvial $(\mathrm{P})$ e da temperatura (T), com ajuste do modelo "wave" isotrópico para o cenário A2, A1B e B1, nos períodos $\mathrm{P} 1, \mathrm{P} 2$ e P3.

Para o cenário A2 (Figura 1), a precipitação apresentou valores máximos próximos de $240 \mathrm{~mm}$, durante o P1, com temperaturas de, aproximadamente, $26^{\circ} \mathrm{C}$. No período seco, o índice pluvial apresentou valores próximos de $10 \mathrm{~mm}$, com temperaturas baixas em torno de $17^{\circ} \mathrm{C}$. Com relação à precipitação, os resultados do $\mathrm{P} 2$ não variaram muito quando comparados aos resultados do $\mathrm{P} 1$, enquanto para temperatura, de 2033 a 2055 , houve valores de $17^{\circ} \mathrm{C}$ e, a partir daí, até 2066 , houve um aumento que varia de $17^{\circ} \mathrm{C}$ a $18^{\circ} \mathrm{C}$; já para o $\mathrm{P} 3$, verificaram-se, pelo mapa, mudanças nos padrões da precipitação e, para temperatura, um aumento que vai de, aproximadamente, $17^{\circ} \mathrm{C}$ até o ano de 2080 , para $18^{\circ} \mathrm{C}$ e $20^{\circ} \mathrm{C}$ até 2098 , no período seco. 
Para o cenário A1B intermediário (Figura 1), a variável temperatura apresentou algumas mudanças em seus períodos, com aumento variando de $17^{\circ} \mathrm{C}$ a $21^{\circ} \mathrm{C}$, no período seco, considerado mais frio. Para o período chuvoso, verificou-se verão mais quente, com precipitação entre 220 a $240 \mathrm{~mm}$, nos três períodos, com mínimas, nos meses de maio a setembro, de 10 a $30 \mathrm{~mm}$, aproximadamente.

No cenário B1 (Figura 1), as mudanças na temperatura e nos padrões de precipitação foram menores quando comparada a dos outros dois cenários. Isso já era esperado, pois esse cenário é o mais otimista quanto às simulações futuras, dando ênfase a soluções globais para a economia e para a sustentabilidade ambiental e social.

A temperatura nos períodos secos variou de $17^{\circ} \mathrm{C}$ a $19^{\circ} \mathrm{C}$, com precipitação mínima de 10 a $30 \mathrm{~mm}$ e, no verão, a temperatura não variou muito, situando-se na faixa de $25^{\circ} \mathrm{C}$ a $26^{\circ} \mathrm{C}$, entre os três períodos (P1, P2 e P3). Para a precipitação, os valores foram de, aproximadamente, $230 \mathrm{~mm}$.

De acordo com os resultados obtidos pela validação cruzada, apresentados na Tabela 3, para as duas variáveis, verificou-se que o modelo "wave" isotrópico foi adequado para representar o padrão temporal analisado, bem como para representar esse padrão pelos mapas de contorno. Isso pode ser explicado pelos resultados dos EM e RQME, os quais, segundo Webster \& Oliver (2001), devem ser próximos de zero e 1 , respectivamente.

Os modelos "wave" isotrópicos apresentaram coeficientes de erros satisfatórios para caracterizar a variabilidade temporal da precipitação pluvial e da temperatura média do ar de Juiz de Fora, nos cenários de mudanças climáticas avaliados.

Tabela 3. Erro médio (EM) e razão do quadrado médio do erro (RQME) para a krigagem ordinária (KO), utilizando-se o modelo "wave", ajustado aos semivariogramas experimentais isotrópicos, referentes à precipitação pluvial (mm) e à temperatura $\left({ }^{\circ} \mathrm{C}\right)$ de Juiz de Fora, para os cenários futuros A2, A1B e B1, nos períodos, P1, P2 e P3.

\begin{tabular}{cccccc}
\hline & & \multicolumn{2}{c}{ Precipitação $(\mathrm{mm})$} & \multicolumn{2}{c}{ Temperatura $\left({ }^{\circ} \mathrm{C}\right)$} \\
\cline { 3 - 5 } & & EM & RQME & EM & RQME \\
\hline \multirow{2}{*}{ A2 } & P1 & 0,254 & 0,817 & 0,000 & 0,589 \\
& P2 & 0,233 & 0,658 & 0,000 & 0,569 \\
& P3 & 0,226 & 0,648 & 0,000 & 0,680 \\
\hline \multirow{2}{*}{ A1B } & P1 & 0,228 & 0,800 & 0,002 & 0,702 \\
& P2 & 0,228 & 0,678 & 0,002 & 0,716 \\
& P3 & 0,207 & 0,792 & 0,002 & 0,746 \\
\hline \multirow{2}{*}{ B1 } & P1 & 0,223 & 0,819 & 0,002 & 0,722 \\
& P2 & 0,240 & 0,875 & 0,002 & 0,722 \\
& P3 & 0,203 & 0,798 & 0,003 & 0,761 \\
\hline
\end{tabular}

Obs.: Cenário pessimista (A2), intermediário (A1B) e otimista (B1), nos períodos de 2000 a 2032 (P1), 2033 a 2065 (P2) e 2066 a 2099 (P3).

Com os resultados dos mapas de krigagem foi possível observar uma aparente regularidade climática para os cenários futuros do IPCC, ou seja, com distribuição interanual das chuvas em sucessão de anos de verões úmidos de outubro a março e invernos secos de abril a setembro. Sant'Anna Neto (2005), ao estudar os dados observados no período de 1969 a 2005, de Presidente Prudente, SP, também verificou tendência de períodos mais secos entre maio a setembro, com irregularidades pluviais entre junho e agosto. O mesmo autor, estudando a temperatura nesse período, verificou mais regularidade para essa variável, ainda que, em alguns anos, os invernos e verões sejam mais rigorosos.

De forma geral, com o uso do interpolador geoestatístico (krigagem ordinária), construíram-se mapas de contorno para os cenários futuros de mudanças climáticas, por meio do arranjo proposto em meses e anos. Com isso, verificaram-se possíveis alterações climáticas em Juiz de Fora, ao longo dos próximos 99 anos, no tocante aos padrões de precipitação e de temperatura do ar no período estudado. Assim, tornou-se evidente que a krigagem pode ser utilizada como projeção para avaliar séries temporais em trabalhos com variáveis climáticas, conforme os resultados obtidos por meio da validação cruzada.

Vários pesquisadores levantaram a hipótese de que as mudanças climáticas que vêm ocorrendo ou que ocorrerão no futuro podem ser devido às atividades antrópicas (Marengo et al., 2007). Verifica-se que o homem pode e vem influenciando o clima, sobretudo com o aumento populacional e o desenvolvimento tecnológico, que ocasionam transformações no espaço. 
Apesar dos resultados observados para a precipitação e para a temperatura nos cenários analisados, caracterizando situações ora pessimistas, ora otimistas, as projeções de mudanças climáticas derivadas dos modelos globais do IPCC - AR3 não são conclusivas e as incertezas ainda são grandes, de acordo com os modelos e regiões consideradas (IPCC, 2001). Além disso, as alterações climáticas são numerosas e podem ocorrer de forma variada em diferentes escalas de tempo e espaço, principalmente em escalas locais (Steinke et al., 2005). Apesar disso, a constante atualização dos cenários pelo IPCC (IPCC, 2007) sugerem aumento da confiança das previsões, à medida que novos dados observados são incorporados na análise dos modelos.

Observou-se, com os resultados obtidos pela krigagem, que este fato pode ser amplamente demonstrado com base em dados de previsão de cenários futuros do IPCC localizados no interior da divisão política do município de Juiz de Fora, de acordo com o arranjo proposto no presente estudo.

\section{Conclusões}

Com o modelo teórico "wave", observou-se ajuste satisfatório para caracterizar os dados de precipitação pluvial e de temperatura do ar, pelo estimador de semivariograma experimental clássico.

Com o uso da metodologia de análise geoestatística, foi possível verificar que a krigagem pode ser utilizada como projeção para mapear a precipitação pluvial e a temperatura do ar de Juiz de Fora, MG ao longo do tempo.

Foi possível verificar, para os cenários A2, A1B e B1, possíveis mudanças nos padrões de precipitação pluvial e temperatura para os dados de previsão de mudanças climáticas, com maior alteração para o cenário mais pessimista (A2).

\section{Agradecimento}

A autora agradece o Grupo de Pesquisa em Modelos Estatísticos, Matemáticos e Fuzzy - MEMF.

\section{Referências}

Andriotti, J. L. S. (2003). Fundamentos de estatıstica e geoestatıstica. Sao Leopoldo, RS: Universidade do Vale do Rio dos Sinos.

Brasil. (1992). Normais Climatologicas (1961-1990). Ministério Da Agricultura e Reforma Agrária. Secretaria Nacional de Irrigação. Departamento Nacional de Meteorologia.

Cambardella, C. A., Moorman, T. B., Parkin, T. B., Karlen, D. L., Novak, J. M., Turco, R. F., \& Konopka, A. E. (1994). Field-scale variability of soil properties in central Iowa soils. Soil Science Society of America Journal, 58(5), 1501-1511.

Chilès, J. P., \& Delfiner, P. (2009). Geostatistics: Modeling Spatial Uncertainty. Wiley. https://books.google.com.br/books?id=tZ107WdjYHgC

Goovaerts, P. (1997). Geostatistics for natural resources evaluation. Oxford University Press on Demand.

IPCC. (2001). Climate Change 2001: The Scientific Basis. In In: Houghton, J. T.; Ding, Y.; Griggs, D. J.; Noguer, N.; Vander Linden, P. J.; Xiaosu, D.; Maskell, K.; Johnson, C. A. (Ed.). (Cambridge:).

IPCC. (2007). Climate Change 2007 - The Physical Science Basis: Working Group I Contribution to the Fourth Assessment Report of the IPCC. Cambridge University Press. https://books.google.com.br/books?id=8m8nXB8GB4C

Marengo, J. A., Alves, L. M., Castro, C. A. C., \& Mendes, D. (2007). Início da estação chuvosa nas Regiões Sudeste, Centro-Oeste e sul do Nordeste do Brasil, afetadas pela crise de energia. Setembro. http://www.cptec.inpe.br/energia/saiba/iniciochuvas.shtml

Mendonça, F., \& Danni-Oliveira, I. M. (2017). Climatologia: noções básicas e climas do Brasil. Oficina de textos.

Mota, V. C. (2008). Metodologia geoestatística para caracterizar a variabilidade temporal de elementos climáticos de Juiz de Fora-MG. Dissertação Mestrado, Universidade Federal de Lavras (UFLA), BRASIL. 
Mota, V. C., Andrade, E. T., \& Leite, D. F. (2019). Caracterização da variabilidade espacial dos índices de conforto animal em sistemas de confinamento Compost Barn. Pubvet, 13(2), 1-14. https://doi.org/10.31533/pubvet.v13n3a276.1-14

Mota, V. C., Lima, R. R., Carvalho Alves, M., Oliveira, M. S., \& Carvalho, L. G. (2008). Dependência temporal da precipitação pluvial e da temperatura do ar em Juiz de Fora, MG, por meio de análises geoestatísticas. Revista Brasileira de Agrometeorologia, 16(3), 249-258.

NCAR. (2007). Geografic Information Sistem (Gis) Initiative. NCAR/UCAR/NSF - NATIONAL CENTER FOR ATMOSPHERIC RESEARCH. http://www.gis.ncar.edu

R-Core-Team. (2016). $R$ : A language and environment for statistical computing. R. Foundation for Satatical Computing.

Ribeiro Júnior, P. J., \& Diggle, P. J. (2001). geoR: A package for geostatistical analysis. R-NEWS, 1 , $15-18$.

Sant'Anna Neto, J. L. (2005). Decálogo da climatologia do Sudeste Brasileiro. Revista Brasileira de Climatologia, 1(1). https://doi.org/10.5380/abclima.v1i1.25232

Soares, E., Mota, V., Poucas, R., \& Leite, D. (2017). Cloud-based evolving intelligent method for weather time series prediction. 2017 IEEE International Conference on Fuzzy Systems (FUZZIEEE), 1-6. https://doi.org/10.1109/FUZZ-IEEE.2017.8015532

Steinke, E. T., Souza, G. A., \& Hiroo Saito, C. H. S. (2005). Análise da variabilidade da temperatura do ar e da precipitação no Distrito Federal no período de 1965/2003 e sua relação com uma possível alteração climática. Revista Brasileira de Climatologia, 1(1). https://doi.org/10.5380/abclima.v1i1.25230

Webster, R., \& Oliver, M. A. (2001). Geostatistics for Environmental Scientists. John Wiley \& Sons Inc.

\section{Histórico do artigo:}

Recebido: 12 de agosto de 2020.

Aprovado: 19 de setembro de 2020.

Disponível online: 12 de novembro de 2020.
Licenciamento: Este artigo é publicado na modalidade Acesso Aberto sob a licença Creative Commons Atribuição 4.0 (CC-BY 4.0), a qual permite uso irrestrito, distribuição, reprodução em qualquer meio, desde que o autor e a fonte sejam devidamente creditados. 